In the book: Spectral Theory and Nonlinear Analysis, World Sci. Publishers, Singapore, 2005, pp. 201-228 (ed. J. Lopez-Gomez) 


\title{
DYNAMICAL SYSTEMS METHOD (DSM) AND NONLINEAR PROBLEMS
}

\author{
A.G. RAMM \\ Mathematics Department, Kansas State University, \\ Manhattan, KS 66506-2602, USA \\ ramm@math.ksu.edu \\ http://www.math.ksu.edu/ ramm
}

\begin{abstract}
.
The dynamical systems method (DSM), for solving operator equations, especially nonlinear and ill-posed, is developed in this paper. Consider an operator equation $F(u)=0$ in a Hilbert space $H$ and assume that this equation is solvable. Let us call the problem of solving this equation illposed if the operator $F^{\prime}(u)$ is not boundedly invertible, and well-posed otherwise. The DSM for solving linear and nonlinear ill-posed problems in $H$ consists of the construction of a dynamical system, that is, a Cauchy problem, which has the following properties:

(1) it has a global solution,

(2) this solution tends to a limit as time tends to infinity,

(3) the limit solves the original linear or non-linear problem.

The DSM is justified for:

(a) an arbitrary linear solvable equations with bounded operator,

(b) for well-posed solvable nonlinear equations with twice Fréchet differentiable operator $F$,

(c) for ill-posed solvable nonlinear equations with monotone operators,

(d) for ill-posed solvable nonlinear equations with non-monotone operators from a wide class of operators,

(e) for ill-posed solvable nonlinear equations with operators $F$ such that $A:=F^{\prime}(u)$ satisfies the spectral assumption of the type $\left\|(A+s I)^{-1}\right\| \leq c / s$, where $c>0$ is a constant, and $s \in\left(0, s_{0}\right), s_{0}>0$ is a fixed number, arbitrarily small, $c$ does not depend on $s$ and $u$,

and
\end{abstract}


(f) for some monotone operators which are not Fréchet differentiable and for some unbounded, closed, densely defined $F$.

In Newton-type schemes the main difficulty is to invert the derivative of the operator. A novel scheme, based on DSM, allows one to avoid this inversion.

Global convergence theorem is obtained for the regularized continuous analog of Newton's method for monotone operators. Global convergence means that convergence is established for an arbitrary initial approximation, not necessarily the one which is sufficiently close to the solution.

A general approach to constructing convergent iterative schemes for solving well-posed nonlinear operator equations is described and convergence theorems are obtained for such schemes.

Stopping rules for stable solution of ill-posed problems with noisy data are given.

\section{Introduction}

The aim of this paper is to present in a self-contained way a series of the results obtained in ${ }^{10} \_22$, where the DSM (dynamical systems method) in its current scope was developed. Among related ideas is the old method of steepest descent. One of the first papers, where the DSM, specifically, its version corresponding to a continuous analog of the Newton's method for well-posed equation $F(u)=0$ was developed, is ${ }^{5}$. In ${ }^{9}$ iterative processes for solving nonlinear well-posed equations in finite-dimensional spaces are presented, and in ${ }^{4}$ iterative methods for solving some ill-posed problems are discussed. In ${ }^{1}{ }^{3},{ }^{7},{ }^{26}$ some results which are obtained by the DSM method are given, and in ${ }^{24}$ and ${ }^{25}$ the efficiency of the DSM method is illustrated in the problem of stable numerical differentiation of noisy data.

The DSM method can be considered as a general method for solving operator equations, especially ill-posed, nonlinear.

At first, one may think that solving the Cauchy problem (4) of the DSM in order to solve equation (1) is replacing a simpler problem (1) by a more complicated problem (4). However, if problem (1) is ill-posed in the sense that (3) fails, then there are no general approaches to solving (1), and the DSM provides such an approach for a very wide class of equations (1).

It also provides a general approach to construction of convergent iterative schemes for solving (1). Finally, numerical solution of (4), after a discretization, amounts to solving a Cauchy problem for a system of ODE. This is an area of numerical analysis which was much studied and is well 
developed.

Let us now describe the idea of the DSM.

Let $H$ be a real Hilbert space, $F: H \rightarrow H$ be an operator. One wants to solve an equation

$$
F(u)=0
$$

and one assumes that there is a $y$, possibly nonunique, such that $F(y)=0$.

Let $u_{0} \in H$ be an element, and $B\left(u_{0}, R\right):=\left\{u:\left\|u-u_{0}\right\| \leq R\right\}$. Assume

$$
\sup _{u \in B\left(u_{0}, R\right)}\left\|F^{(j)}(u)\right\| \leq M_{j}(R), \quad 1 \leq j \leq 2,
$$

where $F^{(j)}$ is the Fréchet derivative. We call problem (1) well-posed, if

$$
\sup _{u \in B\left(u_{0}, R\right)}\left\|\left[F^{\prime}(u)\right]^{-1}\right\| \leq m(R) .
$$

Otherwise we call (1) ill-posed. The dynamical systems method (DSM) for solving (1) is the method consisting of the following steps:

a) finding a map $\Phi(t, u)$ such that the problem

$$
\dot{u}=\Phi(t, u), \quad u(0)=u_{0} ; \quad \dot{u}:=\frac{d u}{d t},
$$

has the following properties:

$$
\exists ! u(t) \quad \forall t>0 ; \quad \exists u(\infty):=\lim _{t \rightarrow \infty} u(t) ; \quad F(u(\infty))=0,
$$

b) solving (4), then taking $t \rightarrow \infty$, and finding the solution as the limit $u(\infty)$.

Note that $u(\infty)=u\left(\infty, u_{0}\right)$ if (1) has more than one solution. How does one find $\Phi$ ? There are many ways $\left({ }^{13}\right)$ to find $\Phi$ such that (5) holds.

We assume that $\Phi$ is locally Lipschitz with respect to $u$ and continuous with respect to $t$. This implies local existence and unqueness of the solution to (4).

Our aim is to review some of the results obtained recently ${ }^{10} \_22$. These results demonstrate the power of the DSM, both as a theoretical tool for proving the existence of a solution to equation (1), proving that $F$ is a global homeomorphism (under suitable additional assumptions), deriving convergent iterative schemes for solving (1), and developing numerical methods for solving a very wide class of linear and nonlinear operator equations, especially ill-posed. There is a large literature on linear ill-posed problems (e.g. see ${ }^{6}$ ), and a less extensive one on nonlinear ill-posed problems (e.g. $\left.27,{ }^{10}\right)$.

Let us describe briefly the scope of the results obtained by the DSM in $10 \_22$, assuming (2) unless otherwise stated: 
(1) Every solvable well-posed equation (1) can be solved by the DSM method which converges exponentially fast.

(2) Every solvable ill-posed linear equation (1) with a bounded operator $A$ can be stably solved by the DSM. Here $F(u):=A u-f=0$, and stably means that if the noisy data $f_{\delta}$ are given, $\left\|f_{\delta}-f\right\| \leq \delta$, then there is a $t_{\delta}$ such that $\lim _{\delta \rightarrow 0}\left\|u\left(t_{\delta}\right)-y\right\|=0$.

(3) Every solvable ill-posed equation (1) with monotone operator $F$, i.e., $(F(u)-F(v), u-v) \geq 0$, can be stably solved by the DSM.

(4) Every solvable equation (1) can be stably solved by the DSM provided that the operator $\widetilde{T}:=\widetilde{A}^{*} \widetilde{A}, \widetilde{A}:=F^{\prime}(y)$, maps the set $\{u:\|u\| \leq r\}$, where $r>0$ is sufficiently small, into the set $\beta:=\{u: 0<\|u\| \leq 1\}$.

(5) If $F$ is monotone, hemicontinuous, defined on all of $H$, but (2) is not assumed, then if equation (1) is solvable, possibly nonuniquely, then its minimal-norm solution $y$ can be stably found by the DSM.

(6) If $F=L+g$, where $L$ is a closed, linear, densely defined in $H$ operator, which has a bounded inverse, $g$ is a nonlinear operator satisfying (2), and equation (1) is solvable, then its solution can be stably found by the DSM, provided, for example, that $\sup _{u \in B\left(u_{0}, R\right)}\left\|\left[I+L^{-1} g^{\prime}(u)\right]^{-1}\right\| \leq m(R)$ and $\sup _{R>0} \frac{R}{m(R)}=\infty$.

(7) A sufficient condition for the surjectivity of a map $F$ is $\sup _{R>0} \frac{R}{m(R)}=\infty$.

(8) A sufficient condition for the map $F$ to be a global homeomorphism is $\left\|\left[F^{\prime}(u)\right]^{-1}\right\| \leq h(\|u\|)$, where $\int^{\infty} \frac{d s}{h(s)}=\infty, h(s)>0$ is a continuous function on $[0, \infty)$.

(9) A method for constructing convergent iterative schemes for solving equation (1) is given.

(10) A method for constructing a Newton-type method without inverting the derivative operator is developed.

\section{Well-posed problems}

Assume (2) and (3). Take $\Phi=-\left[F^{\prime}(u)\right]^{-1} F$ in equation (4):

$$
\dot{u}=-\left[F^{\prime}(u)\right]^{-1} F(u), \quad u(0)=u_{0} .
$$

This is a continuous analog of the Newton's method. From (2) and (3) it follows that $\Phi$ is Lipschitz, so (6) is locally solvable. It will be globally solvable, i.e., solvable $\forall t>0$, if $\sup _{t>0}\|u(t)\| \leq \infty$. Let us prove this estimate. Let $\|F(u(t))\|:=g(t)$. Then $g \dot{g}=-g^{2}$ by $(6)$, so $g(t)=g(0) e^{-t}$. 
From (6) one gets $\|\dot{u}\| \leq g(0) e^{-t} m(R)$. Thus $\|u(t)-u(0)\| \leq g(0) m(R)$ as long as

$$
g(0) m(R) \leq R,
$$

that is, as long as the trajectory $u(t)$ stays in the ball $B\left(u_{0}, R\right)$ for all $t>0$. Assume (7). Then $\exists u(\infty)$,

$$
\sup _{t>0}\left\|u(t)-u_{0}\right\| \leq R, \quad\|u(t)-u(\infty)\| \leq R e^{-t},
$$

and from (6), as $t \rightarrow \infty$, one gets $F(u(\infty))=0$. We have proved that equation (1) can be solved by the DSM (6).

Theorem 2.1. If (2), (3) and (7) hold, then equation (6) has a unique global solution, and (5) holds. Moreover, estimates (8) hold, i.e., u(t) converges to the solution $u(\infty)$ at the exponential rate and the trajectory $u(t)$ stays in the ball $B\left(u_{0}, R\right)$ for all $t \geq 0$.

Remark 2.1. Many other choices of $\Phi$ are discussed in ${ }^{13},{ }^{14}$. These choices include continuous analogs of the modified Newton's method, Gauss-Newton method, gradient method, method of simple iterations, etc.

\section{Surjectivity of $F$}

Theorem 3.1. Assume (2), (3) and let the following condition hold:

$$
\sup _{R>0} \frac{R}{m(R)}=\infty \text {. }
$$

Then $F$ is surjective.

Proof. The proof of Theorem 2.1 shows that if (9) holds, then, for any fixed $u_{0}$, (7) holds for some $R>0$. Thus (1) is solvable. The same argument holds for the equation $F(u)-f=0$, for any $f \in H$. Theorem 3.1 is proved.

\section{When is $F$ a global homeomorphism?}

Theorem 4.1. Assume (2), (3) and let $h: \bar{R}_{+} \rightarrow R_{+}, R_{+}:=(0, \infty)$, be a continuous function such that

$$
\left\|\left[F^{\prime}(u)\right]^{-1}\right\| \leq h(\|u\|), \quad \int_{0}^{\infty} h^{-1}(s) d s=\infty .
$$

Then $F: H \rightarrow H$ is a global homeomorphism. 
Proof. From (3) it follows that $F$ is a local homeomorphism. To prove that $F$ is a global homeomorphism one has to prove that: a) $F$ is surjective, and b) if $F(u)=F(v)$ then $u=v$.

Let us prove a).

As in Section 2, one gets

$$
\frac{d\|u\|}{d t} \leq\|\dot{u}\| \leq h(\|u\|) g_{0} e^{-t}, \quad g_{0}:=g(0),
$$

where the inequality $\|u\|^{\cdot} \leq\|\dot{u}\|$ was used. Thus

$$
\int_{u_{0}}^{\|u(t)\|} \frac{d s}{h(s)} \leq g_{0}\left(1-e^{-t}\right) \leq g_{0}
$$

This and (10) imply $\sup _{t>0}\|u(t)\|<\infty$, so $\sup _{t>0} h(\|u(t)\|)<\infty$, and (11) yields $\|\dot{u}\| \leq c e^{-t}$. Thus $\left\|u(t)-u_{0}\right\| \leq c, u(\infty)$ exists, and, as in Section $2, F(u(\infty))=0$. Since this argument remains valid if $F(u)$ is replaced by $F(u)-f$ with an arbitrary $f \in H$, the surjectivity of $F$ follows. Thus, a) is proved.

Below we consider the equation $F(u)-f=0$.

Let us prove b).

If $\left\|u_{0}-v_{0}\right\|$ is sufficiently small, and

$$
\sup _{t>0}\left\|u\left(t, u_{0}\right)-u\left(t, v_{0}\right)\right\| \leq c\left\|u_{0}-v_{0}\right\|,
$$

where $c>0$ stands for various constants, then b) follows.

Indeed, let $u:=u\left(\infty, u_{0}\right)=\lim _{t \rightarrow \infty} u\left(t, u_{0}\right), F(u)=f=F(v)$, where $v=\lim _{t \rightarrow \infty} v\left(t, v_{0}\right)$. Define $w(s):=u_{0}+s\left(v_{0}-u_{0}\right)$. If $s$ is sufficiently small, then $\left\|w(s)-u_{0}\right\|$ is as small as one wishes, and $\left\|u(t, w(s))-u\left(t, u_{0}\right)\right\|$ is as small as one wishes uniformly for all $t>0$. Thus $\| u(\infty, w(s))-$ $u\left(\infty, u_{0}\right) \|$ is small, $F\left(u\left(\infty, u_{0}\right)\right)=f=F(u(\infty, w(s)))$. Since $F$ is a local homeomorphism, it follows that $u(\infty, w(s))=u\left(\infty, u_{0}\right)=u$. Finitely many small steps are needed to get to $w(1)=v_{0}$ and conclude that $v=$ $u(\infty, w(1))=u\left(\infty, u_{0}\right)=u$.

To complete the proof, let us check (12).

Let $\dot{u}=-\left[F^{\prime}(u)\right]^{-1} F(u), u(0)=u_{0}, \dot{v}=-\left[F^{\prime}(v)\right]^{-1} F(v), v(0)=v_{0}$, $\psi:=u(t)-v(t)$. Then

$$
\dot{\psi}=-\left(\left[F^{\prime}(u)\right]^{-1}-\left[F^{\prime}(v)\right]^{-1}\right)(F(u)-f)-\left[F^{\prime}(v)\right]^{-1}(F(u)-F(v)) .
$$

Let $g(t):=\|\psi(t)\|$. Multiply (13) by $\psi$, use the formula $F(u)-F(v)=$ $F^{\prime}(v)(u-v)+K,\|K\| \leq \frac{M_{2}}{2}\|\psi\|^{2}$, and get

$$
g \dot{g} \leq-g^{2}+c g^{3}+c g^{2} e^{-t},
$$


where the estimate $\|F(u(t))-f\| \leq c e^{-t}$ was used. Since $g(t) \geq 0$, one can consider instead of (14) the following one:

$$
\dot{g} \leq-g+c g^{2}+c g e^{-t}, \quad g(0)=\left\|u_{0}-v_{0}\right\| .
$$

Let $g=e^{-t} h$, so that $g_{0}:=g(0)=h(0)$. Then $\dot{h} \leq c\left(h^{2}+h\right) e^{-t}$. This implies $\ln \frac{h}{h+1} \leq \ln \frac{g_{0}}{g_{0}+1}+c$, i.e., $\frac{h}{h+1} \leq \frac{g_{0}}{g_{0}+1} e^{c}$, where $g_{0}=\|u(0)-v(0)\|$. Therefore, if $g_{0}$ is sufficiently small, so that $g_{0}<\frac{1}{e^{c}-1}$, then $h(t) \leq \operatorname{ch}(0)=$ $c g(0)$. Therefore $g(t) \leq c e^{-t}\|u(0)-v(0)\|$, so (12) follows. Theorem 4.1 is proved.

\section{Linear ill-posed equations}

Assume

$$
F(u)=A u-f=0,
$$

where $A$ is a bounded linear operator, $A y=f, y$ is the minimal-norm solution, $B:=A^{*} A, h:=A^{*} f,\left\|f_{\delta}-f\right\| \leq \delta, \varepsilon(t)>0$ is a monotonically decreasing function, $\lim _{t \rightarrow \infty} \varepsilon(t)=0, \int_{0}^{\infty} \varepsilon(s) d s=\infty$. A solvable equation $A u=f$ is equivalent to

$$
B u=h, \quad B=A^{*} A \geq 0 .
$$

Consider the equation

$$
\dot{u}=-u+[B+\varepsilon(t)]^{-1} h, \quad u(0)=u_{0}, \quad h=A^{*} f .
$$

Theorem 5.1. Any solvable linear equation (16) with $\|A\|<\infty$ can be stably solved by the DSM.

Proof. A solvable linear equation (16) is equivalent to (17): every solution to (16) solves (17) and vice versa. Consider a DSM (18). Since this is a linear equation, clearly $u(t)$ exists for all $t>0$ and is unique. The solution to (18) is: $u(t)=u_{0} e^{-t}+\int_{0}^{t} e^{-(t-s)}[B+\varepsilon(s)]^{-1} h d s$, and, since $h=B u$, it is easy to check that $\lim _{t \rightarrow \infty} u(t)=y$. Indeed, $\lim _{\varepsilon \rightarrow 0}[B+\varepsilon]^{-1} B u=y$, where $u=u(t)$ and $\varepsilon=\varepsilon(t)$, and $\lim _{t \rightarrow \infty} \int_{0}^{t} e^{-(t-s)} g(s) d s=g(\infty)$ provided there exists $g(\infty):=\lim _{t \rightarrow \infty} g(t)$. Thus (5) holds and the DSM (18) is justified. If $f_{\delta}$ is given in place of $f$, then $h_{\delta}$ will replace $h$ in (18). Without loss of generality one may assume that $\left\|h_{\delta}-h\right\| \leq \delta$. Let $u_{\delta}(t)$ solve (18) with $h$ replaced by $h_{\delta}$. Let us prove:

Claim: There exists $t_{\delta}, \lim _{\delta \rightarrow 0} t_{\delta}=\infty$, such that

$$
\lim _{\delta \rightarrow 0}\left\|u_{\delta}\left(t_{\delta}\right)-y\right\|=0 .
$$


To prove the claim, note that $\left\|u_{\delta}\left(t_{\delta}\right)-y\right\| \leq\left\|u_{\delta}\left(t_{\delta}\right)-u\left(t_{\delta}\right)\right\|+\left\|u\left(t_{\delta}\right)-y\right\|$. Since $\lim _{\delta \rightarrow 0}\left\|u\left(t_{\delta}\right)-y\right\|=0$, it is sufficient to prove $\lim _{\delta \rightarrow 0} \| u_{\delta}\left(t_{\delta}\right)-$ $u\left(t_{\delta}\right) \|=0$. Let $u_{\delta}(t)-u(t):=v(t), h_{\delta}-h:=p$. Then

$$
\dot{v}=-v+(B+\varepsilon(t))^{-1} p, \quad v(0)=0 .
$$

Thus

$$
\|v\|=\left\|\int_{0}^{t} e^{-(t-s)}(B+\varepsilon(s))^{-1} p d s\right\| \leq \frac{\delta}{\varepsilon(t)} .
$$

Therefore any $t_{\delta}$ such that $\lim _{\delta \rightarrow 0} t_{\delta}=\infty$ and $\lim _{\delta \rightarrow 0} \frac{\delta}{\varepsilon\left(t_{\delta}\right)}=0$ is suitable for the claim to hold. Theorem 5.1 is proved.

\section{Nonlinear equations with monotone operators}

Assume that $F$ is monotone in the sense

$$
(F(u)-F(v), u-v) \geq 0 \quad \forall u, v \in H,
$$

(2) holds, and

$$
F(y)-f=0,
$$

where $y$ is the minimal-norm solution to (19). Note that if $F$ is monotone and (2) holds, then $N(F):=\{u: F(u)-f=0\}$ is a closed and convex set. Such sets in Hilbert spaces have a unique minimal-norm element, as is well known.

Consider the problem

$$
\dot{u}=-A_{\varepsilon}^{-1}[F(u)+\varepsilon u-f], \quad u(0)=u_{0},
$$

where $A_{\varepsilon}:=F^{\prime}(u)+\varepsilon I, I$ is the identity operator,

$$
\varepsilon=\varepsilon(t)>0, \quad \dot{\varepsilon}(t)<0, \quad \lim _{t \rightarrow \infty} \varepsilon(t)=0, \quad \frac{|\dot{\varepsilon}|}{\varepsilon} \leq \frac{1}{2} .
$$

Theorem 6.1. If (2) and (21) hold, and $u_{0} \in H$ is arbitrary, then (5) holds (with $F(u)-f$ in place of $F(u)$ ) and $u(\infty)=y$. If $f_{\delta}$ replaces $f$ in (19) and (20), $\left\|f_{\delta}-f\right\| \leq \delta$, then there exists $t_{\delta}, \lim _{\delta \rightarrow 0} t_{\delta}=\infty$, such that $\lim _{\delta \rightarrow 0}\left\|u_{\delta}\left(t_{\delta}\right)-y\right\|=0$ where $u_{\delta}(t)$ solves (20) with $f_{\delta}$ in place of $f$.

Proof. Consider the equation

$$
F(V)+\varepsilon(t) V-f=0 .
$$


It is known (e.g. ${ }^{13}$ ) that if $F$ is monotone and (2) and (21) hold, then there exists a unique solution $V=V(t)$ to $(22)$ and

$$
\sup _{t>0}\|V\| \leq\|y\|, \quad\|\dot{V}\| \leq\|y\| \frac{|\dot{\varepsilon}|}{\varepsilon}, \quad \lim _{t \rightarrow \infty}\|V(t)-y\|=0 .
$$

Since $\|u(t)-y\| \leq\|u(t)-V(t)\|+\|V(t)-y\|$, it follows that $\lim _{t \rightarrow \infty} \| u(t)-$ $y \|=0$ provided that $\lim _{t \rightarrow \infty}\|u(t)-V(t)\|=0$. Let

$$
g(t):=\|u(t)-V(t)\|, \quad w:=u(t)-V(t) .
$$

We want to prove the relation: $\lim _{t \rightarrow \infty} g(t)=0$. From (21) and (20) one gets $\dot{w}=-\dot{V}-A_{\varepsilon}^{-1}[F(u)-F(V)+\varepsilon w]$. Moreover, $F(u)-F(V)=A w+K$, where $\|K\| \leq \frac{M_{2} g^{2}}{2}$, by Taylor's formula. Thus $\dot{w}=-w-A_{\varepsilon}^{-1} K-\dot{V}$. Multiply this by $w$ and get $\dot{g} \leq-g+\frac{M_{2}}{2 \varepsilon(t)} g^{2}+\|y\| \frac{|\dot{\varepsilon}|}{\varepsilon}$. Let $\frac{M_{2}}{2}:=c_{0}$, $\|y\|:=c_{1}$. Then

$$
\dot{g} \leq-g+\frac{c_{0}}{\varepsilon(t)} g^{2}+c_{1} \frac{|\dot{\varepsilon}|}{\varepsilon} .
$$

It follows from (24), (21), and Example 7.1 in the next section, that $g(t) \leq$ $c \varepsilon(t) \rightarrow 0$ as $t \rightarrow \infty$. Theorem 6.1 is proved.

We have used in (20) the operator $A_{\epsilon}^{-1}:=(A+\epsilon I)^{-1}$ as an approximation to the inverse of the operator $A:=F^{\prime}(u)$, where $\epsilon>0$ tends to zero. This is natural because $A \geq 0$ is a bounded selfadjoint operator. For such an operator one can derive the relation $\lim _{\epsilon \rightarrow 0} A_{\epsilon}^{-1} A u=u$, using the spectral theorem for selfadjoint operators, and assuming that $u \in D(A), u \perp N(A):=\{u: A u=0\}$, and otherwise $u$ is arbitrary. For non-monotone $F$, and also for monotone $F$, one may use other approximations for the inverse of $A$ when this inverse is unbounded or does not exist. If $A$ is selfadjoint, but not necessarily non-negative, or if $A$ is normal, or, more generally, when $A$ is a spectral operator, then one can use spectral theory to approximate the inverse of $A$. For instance, if $A$ is selfadjoint, then one may use a function $\phi_{\epsilon}(A)$ such that $\lim _{\epsilon \rightarrow 0} \phi_{\epsilon}(A) A u=u$ for all $u \in D(A), u \perp N(A)$. When $A$ is non-selfadjoint, one may use $\phi_{\epsilon}\left(A^{*} A\right)$ to approximate the inverse of $A$, when this inverse is unbounded or does not exist. The regularized Gauss-Newton method is based on such an approximation. A motivation for this is the formula $\left(A^{*} A\right)^{-1} A^{*}=A^{-1}$ valid for a boundedly invertible linear operator $A$.

One can derive a stopping rule for problem (20) as in Section 5. If $\left\|f_{\delta}-f\right\| \leq \delta$, and $u_{\delta}(t)$ solves $(20)$ with $f_{\delta}$ replacing $f$, then the error $E$ 
can be estimated as follows:

$$
E:=\left\|u_{\delta}(t)-y\right\| \leq\left\|u_{\delta}(t)-V_{\delta}(t)\right\|+\left\|V_{\delta}(t)-V(t)\right\|+\|V(t)-y\|,
$$

where $V_{\delta}(t)$ solves $(22)$ with $f_{\delta}$ replacing $f$. One has $\|V(t)-y\|:=a(t) \rightarrow 0$ as $t \rightarrow \infty$. Using monotonicity of $F$, one derives the estimate

$$
\left\|V_{\delta}(t)-V(t)\right\| \leq \frac{\delta}{\varepsilon(t)} .
$$

If $w_{\delta}(t):=u_{\delta}(t)-V_{\delta}(t)$ and $g_{\delta}(t):=\left\|w_{\delta}(t)\right\|$, then, as in the proof of Theorem 6.1, one derives the inequality similar to (24):

$$
\dot{g}_{\delta} \leq-g_{\delta}+\frac{c_{0}}{\varepsilon(t)} g_{\delta}^{2}+\frac{|\dot{\varepsilon}|}{\varepsilon}\left(c_{1}+\frac{\delta}{\varepsilon}\right)
$$

where the last new term comes from the estimates

$$
\left\|\dot{V}_{\delta}\right\| \leq \frac{|\dot{\varepsilon}|}{\varepsilon}\left\|V_{\delta}\right\|, \quad\left\|V_{\delta}\right\| \leq\|V\|+\frac{\delta}{\varepsilon}, \quad \text { and }\|V\| \leq\|y\| .
$$

Applying Theorem 7.1, one gets the inequality $g_{\delta}(t) \leq c \varepsilon(t)$, where $c>0$ is a constant, provided that $t \leq t_{\delta}$, where the stopping time $t_{\delta}$ is found from the equation $\delta^{a}=\varepsilon(t)$, for a fixed $\delta$ and $0<a<1$. This equation has a unique solution $t_{\delta}$ because $\varepsilon$ decays to zero monotonically. Moreover, $t_{\delta} \rightarrow \infty$ as $\delta \rightarrow 0$. The error estimate is:

$$
E \leq g_{\delta}\left(t_{\delta}\right)+\frac{\delta}{\varepsilon\left(t_{\delta}\right)}+a\left(t_{\delta}\right) \rightarrow 0 \quad \text { as } \delta \rightarrow 0,
$$

because, due to the inequality $0<a<1$, one has $\frac{\delta}{\varepsilon\left(t_{\delta}\right)}=\delta^{1-a} \rightarrow 0$ as $\delta \rightarrow 0$.

A novel discrepancy principle for nonlinear equations with monotone operators is formulated and justified in ${ }^{15}$.

\section{A differential inequality}

Theorem 7.1. Let $g(t) \geq 0$ and

$$
\dot{g}(t) \leq-\gamma(t) g+\alpha(t) g^{2}+\beta(t), \quad g(0)=g_{0}, \quad t \geq 0 .
$$

Assume that $\gamma, \alpha$, and $\beta$ are continuous nonnegative functions, and there exists $a \mu(t)>0 \lim _{t \rightarrow \infty} \mu(t)=\infty$, such that

$$
\alpha(t) \leq \frac{\mu(t)}{2}\left[\gamma(t)-\frac{\dot{\mu}(t)}{\mu(t)}\right] ; \quad \beta(t) \leq \frac{1}{2 \mu(t)}\left[\gamma(t)-\frac{\dot{\mu}(t)}{\mu(t)}\right] ; \quad \mu(0) g(0)<1 .
$$


Then every solution $g(t) \geq 0$ to (25) exists for all $t>0$ and

$$
0 \leq g(t)<\frac{1}{\mu(t)} \quad \forall t>0
$$

Proof. Let $h:=g e^{\int_{0}^{t} \gamma(s) d s}$. Then $(25)$ implies

$$
\dot{h} \leq a(t) h^{2}+b(t), \quad h(0)=g_{0},
$$

where

$$
a=\alpha e^{-\int_{0}^{t} \gamma d s}, \quad b=\beta e^{\int_{0}^{t} \gamma d s} .
$$

Consider the equation

$$
\dot{u}=\frac{\dot{f}}{G} u^{2}-\frac{\dot{G}}{f}, \quad u(0)=g_{0} .
$$

Its solution is $\left(\mathrm{cf.}^{8}\right)$ :

$$
u(t)=-\frac{G(t)}{f(t)}+\frac{1}{f^{2}(t)\left[C-\int_{0}^{t} \frac{\dot{f} d s}{G f^{2}}\right]} .
$$

Let

$$
f=\mu^{\frac{1}{2}} e^{-\frac{1}{2} \int_{0}^{t} \gamma d s}, \quad G=-\mu^{-\frac{1}{2}} e^{\frac{1}{2} \int_{0}^{t} \gamma d s} .
$$

Then $C=\frac{1}{\mu(0) g_{0}-1}$. From (26) one gets $a \leq \frac{\dot{f}}{G}, b \leq \frac{\dot{G}}{f}$. Since $f G=-1$, one has $\int_{0}^{t} \frac{\dot{f} d s}{G f^{2}}=\frac{1}{2} \int_{0}^{t}\left(\gamma-\frac{\dot{\mu}}{\mu}\right) d s$, and

$$
u=\frac{e^{\int_{0}^{t} \gamma d s}}{\mu}\left[1-\frac{1}{\frac{1}{1-\mu(0) g(0)}+\frac{1}{2} \int_{0}^{t}\left(\gamma-\frac{\dot{\mu}}{\mu}\right) d s}\right] .
$$

From a comparison lemma it follows that $h \leq u<\frac{e^{\int_{0}^{t} \gamma d s}}{\mu}$, so $g<\frac{1}{\mu} \quad \forall t>0$. Theorem 7.1 is proved.

Example 7.1. Consider an example of applications of Theorem 7.1. This example deals with inequality (24). Let $\mu=\frac{\lambda}{\varepsilon(t)}, \lambda=$ const. Then $\frac{\dot{\mu}}{\mu}=$ $\frac{|\dot{\varepsilon}|}{\varepsilon} \leq \frac{1}{2}$ by (21), so $1-\frac{\dot{\mu}}{\mu} \geq \frac{1}{2}$. Let us check conditions (26). In (24) $\gamma=1$, $\alpha=\frac{c_{0}}{\varepsilon}, \beta=c_{1} \frac{|\dot{\varepsilon}|}{\varepsilon}$. Conditions (26) hold if

$$
\frac{c_{0}}{\varepsilon(t)} \leq \frac{\lambda}{2 \varepsilon} \frac{1}{2}, \quad c_{1} \frac{|\dot{\varepsilon}|}{\varepsilon} \leq \frac{\varepsilon}{2 \lambda} \frac{1}{2}, \quad g(0) \frac{\lambda}{\varepsilon(0)}<1 .
$$

Let $\lambda=4 c_{0}$. Then the first inequality holds. The second one can be written as $4 \lambda c_{1} \frac{|\dot{\varepsilon}|}{\varepsilon^{2}} \leq 1$. A scaling transformation of $\varepsilon$, namely $\varepsilon \mapsto \nu \varepsilon$, 
$\nu=$ const $>0$, leaves $\frac{|\dot{\varepsilon}|}{\varepsilon}$ invariant and transforms $\frac{|\dot{\varepsilon}|}{\varepsilon^{2}}$ into $\frac{1}{\nu} \frac{|\dot{\varepsilon}|}{\varepsilon^{2}}$. Thus, one can choose $\nu$ sufficiently large to satisfy the second inequality (29) and also the third one. By Theorem (25) one gets $0 \leq g(t)<\frac{\varepsilon(t)}{\lambda}=c \varepsilon$, where $c:=\frac{1}{\lambda}>0$.

\section{A spectral assumption and nonlinear equations}

Theorem 8.1. Let (2) hold, $\varepsilon=$ const $>0$, and assume

$$
\left\|A_{\varepsilon}^{-1}(u)\right\| \leq \frac{c}{\varepsilon}, \quad A(u):=A:=F^{\prime}(u), \quad A_{\varepsilon}:=A+\varepsilon I .
$$

Then there exists a solution to the equation:

$$
F(u)+\varepsilon u=0 .
$$

Proof. Consider the problem

$$
\dot{u}=-A_{\varepsilon}^{-1}(u)(F(u)+\varepsilon u), \quad u(0)=u_{0} .
$$

This problem is uniquely locally solvable, because (2) and (30) imply that the right-hand side in (32) is a Lipschitz function. Let

$$
g(t):=\|F(u(t))+\varepsilon u(t)\|,
$$

where $u(t)$ solves $(32)$. Then

$$
g \dot{g}=-\left(A_{\varepsilon} \dot{u}, F(u)+\varepsilon u\right)=-g^{2} .
$$

Thus $g(t)=g_{0} e^{-t}$, and (32) and (30) imply $\|\dot{u}\| \leq \frac{c g_{0}}{\varepsilon} e^{-t}$. Thus $\lim _{t \rightarrow \infty} u(t):=u(\infty)$ exists. Passing to the limit $t \rightarrow \infty$ in (32) one proves that $u(\infty)$ satisfies equation (31). Theorem 8.1 is proved.

\section{A singular perturbation problem}

In this Section we want to give sufficient conditions for the solution $u_{\varepsilon}$ to equation:

$$
F\left(u_{\varepsilon}\right)+\varepsilon\left(u_{\varepsilon}-w\right)=0
$$

to have a limit $\lim _{\varepsilon \rightarrow 0} u_{\varepsilon}=y$, which solves (1). Here $w \in H$ is some element.

Theorem 9.1. Assume: a) (2) with $j \leq 3$ holds, b) $\exists y: F(y)=0, c)(30)$ holds for all $\varepsilon \in\left(0, \varepsilon_{0}\right), \varepsilon_{0}>0$ is a small fixed number, d) $\exists w$ such that $y-w=A v$, where $A:=F^{\prime}(y)$ and $\|v\|<\left[2 M_{2} c(1+c)\right]^{-1}$, where $M_{2}$ is the 
constant from (2) and $c$ is the constant from (30). Then (33) has a solution $u_{\varepsilon}$ for any $\varepsilon \in\left(0, \varepsilon_{0}\right)$, and $\left\|y-u_{\varepsilon}\right\|=O(\varepsilon)$ as $\varepsilon \rightarrow 0$. In a sufficiently small ball $\{u:\|u-y\| \leq R\}, R=O(\varepsilon)$, the solution $u_{\varepsilon}$ is unique.

Remark 9.1. If $\overline{R(A)}=H$, then there exists a $w$ such that $y-w=A v$, $\|v\|$ is arbitrarily small. In fact, such a $w$ exists if $\overline{R_{b}} \cap \stackrel{\circ}{B}(0, r) \neq \emptyset$, where $\stackrel{\circ}{B}(0, r):=\{u: 0<\|u\| \leq r\}, R_{b}:=\{v: v=A u,\|u\| \leq b\}$, and the overbar stands for the closure.

Proof. [Proof of Theorem 9.1] Let $z_{\varepsilon}:=u_{\varepsilon}-y, z_{0}=0, F\left(y+z_{\varepsilon}\right):=\phi\left(z_{\varepsilon}\right)$. Then (33) can be written as $\phi\left(z_{\varepsilon}\right)+\varepsilon z_{\varepsilon}+\varepsilon A v=0$. Write $\phi_{\varepsilon}=A z_{\varepsilon}+K$, where $\|K\| \leq \frac{M_{2}\left\|z_{\varepsilon}\right\|^{2}}{2}$. Then

$$
z_{\varepsilon}=-A_{\varepsilon}^{-1} K-\varepsilon A_{\varepsilon}^{-1} A v:=T\left(z_{\varepsilon}\right) .
$$

The map $T$ is a contraction on $B(R):=\{z:\|z\| \leq R\}$, where $R=O(\varepsilon)$. Indeed, $\|T z\| \leq \varepsilon\|v\|+\frac{c}{\varepsilon} \frac{M_{2}}{2}\|z\|^{2} \leq \varepsilon(1+c)\|v\|+\frac{c M_{2}}{2 \varepsilon} R^{2} \leq R$, provided that

$$
R=\frac{\varepsilon}{c M_{2}}(1-\rho),
$$

where $\rho=\sqrt{1-2 M_{2} c(1+c)\|v\|}$. Here we have used the estimate $\left\|A_{\varepsilon}^{-1} A v\right\| \leq\|v\|+\varepsilon\left\|A_{\varepsilon}^{-1} v\right\| \leq(1+c)\|v\|$. If $\|v\|<\frac{1}{2 M_{2} c(1+c)}$, then $0<\rho<1, R=O(\varepsilon)$, and $T B(R) \subset B(R)$. Furthermore, $\|T z-T p\|=$ $\left\|-A_{\varepsilon}^{-1}[K(z)-K(p)]\right\|$, where $z, p \in B(R)$. By Taylor's formula one gets $K(z)=\int_{0}^{1}(1-s) F^{\prime \prime}(y+s z) z z d s$. Thus

$$
\|K(z)-K(p)\| \leq\left\|\int_{0}^{1} d s(1-s)\left[F^{\prime \prime}(y+s z) z z-F^{\prime \prime}(y+s p) p p\right]\right\| .
$$

One has

$$
\begin{gathered}
\left\|F^{\prime \prime}(y+s z) z z-F^{\prime \prime}(y+s p) z z+F^{\prime \prime}(y+s p)(z z-p p)\right\| \\
\leq M_{3} s\|z-p\| R^{2}+2 M_{2} R\|z-p\| .
\end{gathered}
$$

Thus

$$
\|T(z)-T(p)\| \leq q\|z-p\|, \quad q=\frac{c}{\varepsilon}\left(\frac{M_{3} R^{2}}{6}+M_{2} R\right) .
$$

Therefore $T$ maps $B(R)$ into itself and is a contraction on $B(R)$, where $R$ is defined in (35). Indeed

$$
q=\frac{c}{\varepsilon}\left[\frac{M_{3}}{6} \frac{\varepsilon^{3}}{c^{3} M_{2}^{3}}(1-\rho)^{3}+M_{2} \frac{\varepsilon}{c M_{2}}(1-\rho)\right]=1-\rho+O\left(\varepsilon^{2}\right)<1,
$$


if $\varepsilon$ is sufficiently small. For such $\varepsilon$ equations (34) and (33) are uniquely solvable and $\left\|z_{\varepsilon}\right\| \leq O(\varepsilon)$ as claimed. Theorem 9.1 is proved.

Remark 9.2. Theorem 9.1 holds if $w=0$ and

$$
y=A v,\|v\|<\left[2 M_{2} c(1+c)\right]^{-1} \text {. }
$$

\section{Nonsmooth monotone operators}

Assumption A): Assume that $F$ is hemicontinuous (that is, $(F(u+\lambda h), w)$ is a continuous function of $\lambda$ in a neighborhood of $\lambda=0$ for any $u, h$ and $w)$, monotone: $(F(u)-F(v), u-v) \geq 0 \forall u, v \in H$, defined on all of $H$, and $\exists y: F(y)=0$, where $y$ is the minimal-norm solution to (1).

It is known that under Assumption A) the set $N_{F}:=\{z: F(z)=0\}$ is closed and convex, and such a set in $H$ has a unique element of minimal norm, so $y$ is uniquely defined if (1) has a solution.

Consider the problem:

$$
\dot{u}=-[F(u)+\varepsilon u], \quad u(0)=u_{0}, \quad \varepsilon=\text { const }>0 .
$$

Lemma 10.1. Problem (38) has a unique global solution if A) holds.

This lemma is known (see e.g. ${ }^{10}$ ) and holds also for any integrable $\varepsilon=\varepsilon(t) \geq 0$.

Lemma 10.2. The solution to (38) satisfies the estimates

$$
\sup _{t \geq 0}\|u(t)\| \leq c<\infty ; g(t) \leq g(0) e^{-\varepsilon t}, g(t):=\|u(t+h)-u(t)\|, t \geq 0
$$

where $c=$ const $>0$ and $h=$ const $>0$ is arbitrary.

Proof. Let $z:=u(t+h)-u(t),\|z\|:=g(t)$. From (38) one gets

$$
\dot{z}=-[F(u(t+h))-F(u(t))]-\varepsilon z, \quad z(0)=u(h)-u_{0} .
$$

Multiply this equation by $z$, use the monotonicity of $F$ and get $g \dot{g} \leq-\varepsilon g^{2}$. So $g \leq g(0) e^{-\varepsilon t}$. Let us prove the first inequality (39). Denote $u(t)-y:=p$, $\|p\|:=q$. Then

$$
\dot{p}=-[F(u)-F(y)]-\varepsilon(u-y)-\varepsilon y .
$$

Multiply (41) by $p$, use the monotonicity of $F$ and the inequality $q \geq 0$, and get

$$
\dot{q} \leq-\varepsilon q+\varepsilon\|y\|, \quad q(0)=\left\|u_{0}-y\right\| .
$$


This implies $q(t) \leq q(0) e^{-\varepsilon t}+\varepsilon\|y\| \int_{0}^{t} e^{-\varepsilon(t-s)} d s$. Thus $q(t) \leq\left\|u_{0}-y\right\|+\|y\|$, and the first inequality (39) is proved.

Remark 10.1. We claim that the first inequality (39) holds also if $\varepsilon=\varepsilon(t)>0$, where $\varepsilon(t)$ is a locally integrable function. Indeed, since $q \geq 0$, write $(42)$ as $\dot{q} \leq-\varepsilon q+\varepsilon\|y\|$. Let $q=Q(t) e^{-\int_{0}^{t} \varepsilon(s) d s}$. Then $\dot{Q} \leq\|y\| e^{\int_{0}^{t} \varepsilon(s) d y} \varepsilon(t)$, so $Q(t) \leq Q(0)+\|y\| \int_{0}^{t} \varepsilon(s) e^{\int_{0}^{s} \varepsilon d \tau} d s \leq Q(0)+$ $\|y\| e^{\int_{0}^{t} \varepsilon(s) d s}$. Thus $q(t) \leq q(0)+\|y\|$, and the claim follows.

Theorem 10.1. If $A$ ) holds and $\lim _{\varepsilon \rightarrow 0} \varepsilon t_{\varepsilon}=\infty$, then

$$
\lim _{\varepsilon \rightarrow 0}\left\|u\left(t_{\varepsilon}\right)-y\right\|=0 .
$$

Proof. If A) holds, then equation (31) is uniquely solvable. Denote by $V_{\varepsilon}$ its solution. Then $V_{\varepsilon}$ satisfies the first and the third relation (23). Let $u(t)-V_{\varepsilon}:=\eta$, where $u(t)$ solves problem (38). Then (38) implies

$$
\dot{\eta}=-[F(u)-F(V)+\varepsilon(u-V)] .
$$

Multiply this equation by $\eta$, let $\|\eta\|:=\varphi$, use the monotonicity of $F$, and get $\dot{\varphi} \varphi \leq-\varepsilon \varphi^{2}$. Thus, $\varphi(t) \leq \varphi(0) e^{-\varepsilon t}$. If $t=t_{\varepsilon}$ and $\lim _{\varepsilon \rightarrow 0} \varepsilon t_{\varepsilon}=\infty$, then

$$
\lim _{\varepsilon \rightarrow 0}\left\|u\left(t_{\varepsilon}\right)-V_{\varepsilon}\right\| \leq \varphi(0) \lim _{\varepsilon \rightarrow 0} e^{-\varepsilon t_{\varepsilon}}=0
$$

Since

$$
\|u(t)-y\| \leq\left\|u(t)-V_{\varepsilon}\right\|+\left\|V_{\varepsilon}-y\right\|,
$$

one concludes from (44) and (23) that $\lim _{\varepsilon \rightarrow 0}\left\|u\left(t_{\varepsilon}\right)-y\right\|=0$. Theorem 10.1 is proved.

Assumption B: Assume that the function $\varepsilon(t)>0$ is continuous, differentiable, monotonically decaying to zero as $t \rightarrow \infty$, and

$$
\int_{0}^{\infty} \varepsilon(s) d s=\infty, \quad \lim _{t \rightarrow \infty} \frac{|\varepsilon \dot{\varepsilon(t)}|}{\varepsilon(t)^{2}}=0 .
$$

Theorem 10.2. If $A$ ) and B) hold, then equation

$$
\dot{u}=-F(u)-\varepsilon(t) u, \quad u(0)=u_{0},
$$

has a unique solution, (5) holds, and $u(\infty)=y$. 
Proof. Lemma 10.1 holds for problem (47) as well. If B) holds, then (23) holds with $V=V_{\varepsilon(t)}$. Thus, by (45), if one proves

$$
\lim _{t \rightarrow \infty}\|u(t)-V(t)\|=0,
$$

then Theorem 10.2 is proved. Let $\eta:=u(t)-V(t),\|\eta\|:=\varphi(t)$. From (47) one gets as above, $\dot{\varphi} \leq-\varepsilon(t) \varphi+\frac{|\dot{\varepsilon}|}{\varepsilon}\|y\|$. Thus

$$
\varphi(t) \leq \varphi(0) e^{-\int_{0}^{t} \varepsilon(s) d s}+e^{-\int_{0}^{t} \varepsilon d s} \int_{0}^{t} e^{\int_{0}^{s} \varepsilon d \tau} \frac{|\dot{\varepsilon}|}{\varepsilon}\|y\| d s \rightarrow 0 \quad \text { as } \quad t \rightarrow \infty,
$$

by (46), as one checks using L'Hospital's rule, for example. Theorem 10.2 is proved.

\section{Unbounded operators}

Consider equation (1) with $F=L+g$, where $L$ is a linear, densely defined, closed operator, and $g$ is a nonlinear operator satisfying (2). For example, a semilinear boundary-value problem

$$
-\Delta u+g(u)=f \quad \text { in } \quad D,\left.\quad u\right|_{S}=0
$$

is of this form, $L$ is the Dirichlet operator $-\Delta, H=L^{2}(D)$.

Theorem 11.1. Assume that $\left\|L^{-1}\right\| \leq m$ and

$$
\begin{gathered}
\sup _{u \in B\left(u_{0}, R\right)}\left\|\left[I+L^{-1} g^{\prime}(u)\right]^{-1}\right\| \leq m_{1}, \\
\left\|u_{0}+L^{-1} g^{\prime}\left(u_{0}\right)\right\| m_{1} \leq R .
\end{gathered}
$$

Then (5) holds for problem (4) with $\Phi:=-\left[I+L^{-1} g^{\prime}(u)\right]^{-1}\left[u+L^{-1} g(u)\right]$.

Theorem 11.2. Assume that $L=L^{*} \geq 0, F=L+g, g^{\prime}(u) \geq 0 \forall u \in H, g$ satisfies (2), equation (1) is solvable, $y$ is its minimal-norm solution, $L_{\varepsilon}:=$ $L+\varepsilon I$, and $\Phi:=\Phi_{\varepsilon}:=-\left[I+L_{\varepsilon}^{-1} g^{\prime}(u)\right]^{-1}\left[u+L_{\varepsilon}^{-1} g(u)\right], \varepsilon=$ const $>0$. Suppose (51) holds with $L$ replaced by $L_{\varepsilon}$, and $m_{1}=m_{1}(\varepsilon)>0$. Then (5) holds for (4) and

$$
\lim _{\varepsilon \rightarrow 0}\left\|v_{\varepsilon}-y\right\|=0,
$$

where $v_{\varepsilon}$ solves the equation $L_{\varepsilon} v_{\varepsilon}+g\left(v_{\varepsilon}\right)=0$. 
Proof. [Proof of Theorem 11.1.] Equation (1) is equivalent to

$$
u+L^{-1} g(u)=0 .
$$

Assumptions (51), (52), and Theorem 9.1 imply the conclusion of Theorem 2.1 .

Proof. [Proof of Theorem 11.2.] Consider the equation

$$
L_{\varepsilon} u+g(u)=0, \quad u=u_{\varepsilon} .
$$

By Theorem 11.1 equation (4) with $\Phi=\Phi_{\varepsilon}$ satisfies (5). Let $u_{\varepsilon}(\infty):=v_{\varepsilon}$ solve equation (55). Let us prove (53). Let $v_{\varepsilon}-y:=w_{\varepsilon}$. Then $L w_{\varepsilon}+\varepsilon v_{\varepsilon}+$ $g\left(v_{\varepsilon}\right)-g(y)=0$. Multiplying by $w_{\varepsilon}$ and using the monotonicity of $g$ and $L$, one gets $\varepsilon\left(v_{\varepsilon}, w_{\varepsilon}\right) \leq 0$. Thus

$$
\left\|v_{\varepsilon}\right\| \leq\|y\| .
$$

Thus, there is a weakly convergent subsequence $v_{n}:=v_{\varepsilon_{n}} \rightarrow v_{0}, w_{n}:=$ $v_{n}-y \rightarrow w_{0}:=v_{0}-y$ as $\varepsilon_{n} \rightarrow 0$, and

$$
\limsup _{n \rightarrow \infty}\left\|v_{n}\right\| \leq\|y\|, \quad \liminf _{n \rightarrow \infty}\left\|v_{n}\right\| \geq\left\|v_{0}\right\| .
$$

Let us check that $v_{0} \in D(L)$ and pass to the limit $\varepsilon_{n} \rightarrow 0$ in (55). Since $L$ is selfadjoint one has

$$
(\eta, \psi)=\lim _{n \rightarrow \infty}\left(L v_{n}, \psi\right)=\lim _{n \rightarrow \infty}\left(v_{n}, L \psi\right)=\left(v_{0}, L \psi\right) \quad \forall \psi \in D(L),
$$

where

$$
\eta=\lim _{n \rightarrow \infty} L v_{n}
$$

This strong limit exists because of (55). Indeed, $\lim _{n \rightarrow \infty} \varepsilon_{n} v_{n}=0$ since $v_{n}$ is bounded (see (56)). Let us check that

$$
\lim _{n \rightarrow \infty} v_{n}=v_{0} \text {. }
$$

If (60) holds then $\lim _{n \rightarrow \infty} g\left(v_{n}\right)=g(v)$ by the continuity of $g$, and (59) follows from (55). To prove (60), we first prove that $v_{0}=y$. Assuming this for a moment, one has from (57): $\|y\| \leq \liminf _{n \rightarrow \infty}\left\|v_{n}\right\| \leq$ $\lim \sup _{n \rightarrow \infty}\left\|v_{n}\right\| \leq\|y\|$. Therefore (60) holds with $v_{0}=y$.

Let us prove $v_{0}=y$. One has by the monotonicity:

$$
\left(L v_{n}+g v_{n}+\varepsilon_{n} v_{n}-L\left(v_{0}-t z\right)-g\left(v_{0}-t z\right)-\varepsilon\left(v_{0}-t z\right), v_{n}-v_{0}+t z\right) \geq 0 \text { (61) }
$$

where $t>0$ and $z \in D(L)$ is arbitrary. Since $v_{n} \rightarrow v_{0}$ and $t=$ const $>0$, one gets from (61) as $n \rightarrow \infty$ the following:

$$
\left(-L v_{0}+t L z-g\left(v_{0}-t z\right), z\right) \geq 0 .
$$


Let $t \rightarrow 0$ in (62). Then

$$
\left(-L v_{0}-g\left(v_{0}\right), z\right) \geq 0 \quad \forall z \in D(L) .
$$

Since $D(L)$ is dense in $H$, it follows from (63) that

$$
L v_{0}+g\left(v_{0}\right)=0 \text {. }
$$

However, $\left\|v_{0}\right\| \leq\|y\|$, and $y$ is the minimal-norm solution to (64). Since such a solution is unique, it follows that $v_{0}=y$, as claimed. The uniqueness of the minimal-norm solution to (64) follows from Lemma 11.1.

Lemma 11.1. If $F$ is closed, hemicontinuous, and monotone, and $D(F)$ is a dense linear set, then $N_{F}=\{u: F(u)=0\}$ is a closed and convex set.

Proof. First let us prove that $N_{F}$ is closed. Let $F\left(u_{n}\right)=0, u_{n} \rightarrow u$. Then, since $F$ is closed, one gets $F(u)=0$. So $N_{F}$ is closed. Now let us prove that $N_{F}$ is convex. Note that under the assumptions of Lemma 11.1 one has:

$$
u \in N_{F} \Leftrightarrow(-F(v), v-u) \geq 0 \quad \forall v \in D(F) .
$$

Indeed, if $u \in N_{F}$ then the monotonicity of $F$ implies inequality (65). Conversely, if inequality (65) holds, then set $v=u+t z$, where $t=$ const $>$ 0 and $z \in D(F)$ is arbitrary, and get $-(F(u+t z), z) \geq 0$. Pass to the limit $t \rightarrow 0$ using the hemicontinuity of $F$ and get $-(F(u), z) \geq 0 \forall z \in D(F)$. Since $D(F)$ is dense in $H$, this implies $F(u)=0$. Lemma 11.1 is proved.

In Hilbert spaces any convex and closed set has a unique minimal-norm element.

From our argument and from (55) it follows that $L v_{n} \rightarrow \eta, v_{n} \rightarrow y$, so $L y=\eta$ because $L$ is closed. Theorem 11.1 is proved.

\section{Equations in Banach spaces}

Consider equation (31) in a Banach space $X$. Assume that $\varepsilon=$ const $>0$, $F: X \rightarrow X,(2)$ and (30) hold, and consider problem (32). Let $\eta \in X^{*}$ be arbitrary, $\varphi(t):=\langle F(u(t))+\varepsilon u(t), \eta\rangle, g(t):=\|F(u(t))+\varepsilon u(t)\|=$ $\sup _{\|\eta\| \leq 1}|\varphi(t)|$. Using $(32)$ one gets $\stackrel{\circ}{\varphi}=-\varphi$, so $\varphi(t)=\varphi(0) e^{-t}$. Thus $g(t) \leq g(0) e^{-t}$ and, by (32), $\|\dot{u}\| \leq \frac{c}{\varepsilon} g(0) e^{-t}$. Thus $u(\infty)$ exists, and passing to the limit $t \rightarrow \infty$ in (32) one proves that $u(\infty)$ solves (31).

We have proved that equation (31) can be solved by the DSM:

Theorem 12.1. If $F: X \rightarrow X$, (2) and (30) hold, $\varepsilon=$ const $>0$, then equation (31) has a solution, and (5) holds for (32). 
The proof of Theorem 9.1 remains valid for equation (33) in Banach spaces.

\section{Iterative processes for well-posed problems}

In this section we prove that any solvable well-posed problem can be solved by an iterative process with constant stepsize and the process converges exponentially fast.

Lemma 13.1. Assume:

i) $\left(F^{\prime}(u) \Phi(t, u), F(u)\right) \leq-g_{1}(t)\|F(u)\|^{2} \forall u \in B\left(u_{0}, R\right), \int_{0}^{\infty} g_{1} d t=\infty$,

ii) $\|\Phi(t, u)\| \leq g_{2}(t)\|F(u)\|, \forall u \in B\left(u_{0} R\right)$;

and

iii) $\left\|F\left(u_{0}\right)\right\| \int_{0}^{\infty} G(t) d t \leq R$, where $g_{1}$ and $g_{2}$ are positive integrable functions, $G(t):=g_{2}(t) e^{-\int_{0}^{t} g_{1} d s} \in L^{1}(0, \infty)$, and (4) has a unique local solution.

Then (5) holds for problem (4).

Proof. Since there is a unique solution to (4), this solution exists globally if $\sup _{t>0}\|u(t)\|<\infty$. Let $g(t)=\|F(u(t))\|$. Using (4) one gets $\dot{g} g=\left(F^{\prime} \dot{u}, F\right)=\left(f^{\prime} \Phi, F\right) \leq-g_{1}(t) g^{2}$. Since $g \geq 0$, one obtains $g(t) \leq g(0) e^{-\int_{0}^{t} g_{1} d s}, g(0)=\left\|F\left(u_{0}\right)\right\|$. From (4) it follows that $\|\dot{u}\| \leq g_{2}(t) g(0) e^{-\int_{0}^{t} g_{1} d s}=g(0) G(t) \in L^{1}(0, \infty)$. Therefore $u(\infty)$ exists,

$$
\|u(t)-u(0)\| \leq g(0) \int_{0}^{\infty} G(s) d s \leq R
$$

and

$$
\|u(t)-u(\infty)\| \leq g(0) \int_{t}^{\infty} G(s) d s .
$$

Lemma 13.1 is proved.

Remark 13.1. If $g_{1}=c_{1}=$ const $>0$, and $g_{2}=c_{2}=$ const $>0$, then $\int_{0}^{\infty} G d s=\frac{c_{2}}{c_{1}}$, iii) takes the form $\left\|F\left(u_{0}\right)\right\| \frac{c_{2}}{c_{1}}:=r \leq R$, and one has

$$
\|u(t)-u(\infty)\| \leq r e^{-c_{1} t}, \quad\|F(u(t))\| \leq\left\|F\left(u_{0}\right)\right\| e^{-c_{1} t} .
$$

Theorem 13.1. Assume (2), (3), $\left\|\Phi^{\prime}(u)\right\| \leq L_{1} \forall u \in B\left(u_{0}, R\right)$, and let $F(y)=0$. Let the conditions $i)$, ii), and iii) of Lemma 13.1 hold with $g=c_{j}, j=1,2, g=$ const $>0$, and $u_{0}$ is sufficiently close to $y$. Then there exists an $h>0$ such that the iterative process

$$
u_{n+1}=u_{n}+h \Phi\left(u_{n}\right), \quad u_{0}=u_{0},
$$


converges to $y$ :

$\left\|u_{n}-y\right\| \leq r e^{-\operatorname{chn}}, \quad\left\|F\left(u_{n}\right)\right\| \leq\left\|F_{0}\right\| e^{-\operatorname{chn}}, \quad\left\|F_{0}\right\|:=\left\|F\left(u_{0}\right)\right\|$,

where $c=$ const, $0<c<c_{1}$, and $r:=\frac{c_{2}}{c_{1}}\left\|F_{0}\right\|$.

Proof. For $n=0$ the inequalities (68) hold (see (66) with $u(\infty)=y$ ). We prove that if they hold for $n$ then they hold for $n+1$. The assumption that $u_{0}$ is sufficiently close to $y$ ensures that $y=u(\infty)$.

Let $w_{n+1}(t)$ solve $(4)$ for $t>t_{n}, w_{n+1}\left(t_{n}\right)=u_{n}, t_{0}=0, t_{n}=n h$. Вy Lemma 13.1 one has

$$
\left\|w_{n+1}(t)-y\right\| \leq \frac{c_{2}}{c_{1}}\left\|F_{n}\right\| e^{-c_{1} t} \leq r e^{-c h n-c_{1} t}, \quad t_{n}<t \leq t_{n+1},
$$

where $F_{n}:=F\left(u_{n}\right)$. Also,

$$
\left\|u_{n+1}-y\right\| \leq\left\|u_{n+1}-w_{n+1}\left(t_{n+1}\right)\right\|+\left\|w_{n+1}\left(t_{n+1}\right)-y\right\|,
$$

and

$$
\begin{aligned}
\left\|u_{n+1}-w_{n+1}\left(t_{n+1}\right)\right\| & \leq \int_{t_{n}}^{t_{n+1}}\left\|\Phi\left(u_{n}\right)-\Phi\left(w_{n+1}(s)\right)\right\| d s \\
& \leq L_{1} c_{2} h \int_{t_{n}}^{t_{n+1}} \| F\left(w_{n+1}(s) \| d s\right. \\
& \leq L_{1} c_{1} h^{2} r e^{-c h n} .
\end{aligned}
$$

From (70) and (71) one gets

$$
\left\|u_{n+1}-y\right\| \leq r e^{-c h n}\left(e^{-c_{1} h}+c_{1} L_{1} h^{2}\right) \leq r e^{-c h(n+1)},
$$

provided that $h$ is so small that

$$
e^{-c_{1} h}+c_{1} L_{1} h^{2} \leq e^{-c h}, \quad 0<c<c_{1} .
$$

In (72) we have used the estimate (cf (68)):

$$
\left\|F\left(w_{n+1}(s)\right)\right\| \leq\left\|F_{n}\right\| e^{-c_{1}\left(s-t_{n}\right)} \leq\left\|F_{0}\right\| e^{-\operatorname{chn}-c_{1}\left(s-t_{n}\right)} .
$$

Let us check the second inequality (68) with $n+1$ in place of $n$ :

$$
\left\|F\left(u_{n+1}\right)\right\| \leq\left\|F\left(u_{n+1}\right)-F\left(w_{n+1}\left(t_{n+1}\right)\right)\right\|+\left\|F\left(w_{n+1}\left(t_{n+1}\right)\right)\right\| .
$$

One has

$$
\begin{aligned}
& \left\|F\left(u_{n+1}\right)-F\left(w_{n+1}\left(t_{n+1}\right)\right)\right\| \\
& \quad \leq M_{1}\left\|u_{n+1}-w_{n+1}\left(t_{n+1}\right)\right\| \leq M_{1} L_{1} c_{1} h^{2} r e^{-c h n},
\end{aligned}
$$

where (71) was used. From (76), (74), and (75) one gets:

$$
\left\|F\left(u_{n+1}\right)\right\| \leq\left\|F_{0}\right\| e^{-c h n}\left(M_{1} L_{1} h^{2} c_{2}+e^{-c_{1} h}\right) \leq\left\|F_{0}\right\| e^{-\operatorname{ch}(n+1)},
$$


provided that

$$
M_{1} L_{1} h^{2} c_{2}+e^{-c_{1} h} \leq e^{-c h}, \quad 0<c<c_{1} .
$$

If $h$ is sufficiently small, then (73) and (78) hold. Theorem 13.1 is proved.

\section{Iterative process for ill-posed problems with monotone operators}

Assume (2), but not (3). Let $A:=F^{\prime}(u) \geq 0 \forall u, A_{\varepsilon}:=A+\varepsilon I$, and consider an iterative process

$$
u_{n+1}=u_{n}-h_{n} A_{n}^{-1}\left[F\left(u_{n}\right)+\varepsilon_{n} u_{n}\right], \quad u_{0}=u_{0},
$$

where $A_{n}:=A\left(u_{n}\right)+\varepsilon_{n} I$, and $u_{0} \in H$ is arbitrary. Let $y$ be the minimal norm solution to (1).

Theorem 14.1. Under the above assumptions one can choose $\varepsilon_{n}$ and $h_{n}$ so that

$$
\lim _{n \rightarrow \infty}\left\|u_{n}-y\right\|=0 .
$$

Proof. Let $V_{n}$ solve (22) with $\varepsilon(t)=\varepsilon_{n}$. Let $z_{n}:=u_{n}-V_{n},\left\|z_{n}\right\|=g_{n}$. Then $\left\|u_{n}-y\right\| \leq g_{n}+\left\|V_{n}-y\right\|$, and we know that $\lim _{n \rightarrow \infty}\left\|V_{n}-y\right\|=0$ if $\lim _{n \rightarrow \infty} \varepsilon_{n}=0$. Thus (80) holds if $\lim _{n \rightarrow \infty} g_{n}=0$. Let us prove the last relation. Let $b_{n}:=\left\|V_{n+1}-V_{n}\right\|, \lim _{n \rightarrow \infty} b_{n}=0$. Rewrite (79) as

$$
z_{n+1}=\left(1-h_{n}\right) z_{n}-h_{n} A_{n}^{-1} K\left(z_{n}\right)-\left(V_{n+1}-V_{n}\right),
$$

where we have used the Taylor formula:

$$
\begin{gathered}
F\left(u_{n}\right)+\varepsilon_{n} u_{n}=F\left(u_{n}\right)-F\left(V_{n}\right)+\varepsilon z_{n}=A_{n} z_{n}+K\left(z_{n}\right), \\
\left\|K\left(z_{n}\right)\right\| \leq \frac{M_{2} g_{n}^{2}}{2}:=c g_{n}^{2} .
\end{gathered}
$$

From (81) one gets

$$
g_{n+1} \leq\left(1-h_{n}\right) g_{n}+\frac{c h_{n}}{\varepsilon_{n}} g_{n}^{2}+b_{n}, \quad 0<h_{n} \leq 1 .
$$

Let

$$
\varepsilon_{n}=2 c g_{n}
$$

Then (82) yields:

$$
g_{n+1} \leq\left(1-a_{n}\right) g_{n}+b_{n}, \quad 0<a_{n}=\frac{h_{n}}{2} \leq 0.5 .
$$


Theorem 14.1 follows from Lemma 14.1, which is stated and proved below.

Lemma 14.1. If $b_{n} \geq 0, \lim _{n \rightarrow \infty} b_{n}=0,0<a_{n} \leq \frac{1}{2}$ and

$$
\sum_{n=1}^{\infty} a_{n}=\infty, \quad \lim _{n \rightarrow \infty} \sum_{k=1}^{n-1} b_{k} e^{-\sum_{j=k+1}^{n} a_{j}}=0,
$$

then (84) implies

$$
\lim _{n \rightarrow \infty} g_{n}=0
$$

Proof. From (84) one gets by induction:

$$
g_{n+1} \leq b_{n}+\sum_{k=1}^{n-1} b_{k} \prod_{j=k+1}^{n}\left(1-a_{j}\right)+\prod_{j=1}^{n}\left(1-a_{j}\right) g_{1}
$$

If $a \geq 0$, then $1-a \leq e^{-a}$. Thus $\prod_{j=k+1}^{n}\left(1-a_{j}\right) \leq e^{-\sum_{j=k+1}^{n} a j}$. Therefore (85) and (87) imply (86). Lemma 14.1 is proved.

Remark 14.1. One can always choose $a_{n}$ such that (85) holds. Indeed, let $a_{j}=\log p_{j}=\log p \forall j$, where $1<p \leq \sqrt{e}$. Then $e^{-\sum_{j=k+1}^{n} a_{j}}=p^{-n+k}$, and $\lim _{n \rightarrow \infty} \sum_{k=1}^{n-1} b_{k} p^{-n+k}=0$ if $\lim _{k \rightarrow \infty} b_{k}=0$.

\section{Newton-type methods without inverting the derivative}

In using Newton-type methods the most difficult part of solving numerically problem (1). This part consumes major computer time and leads to numerical errors. Let us consider a DSM of the form

$$
\begin{gathered}
\dot{u}=-Q F, \quad u(0)=u_{0}, \\
\dot{Q}=-T Q+A^{*}, \quad Q(0)=Q_{0},
\end{gathered}
$$

where $\quad A:=F^{\prime}(u), \quad T:=A^{*} A$.

Therefore, instead of an unknown $u$ we are looking for a two-component vector $\left(\begin{array}{c}u \\ Q\end{array}\right)$, where $Q$ is an operator-function which plays the role of $\left[F^{\prime}(u)\right]^{-1}$ in the usual Newton-type method. We prove that problem (88)(89) has a unique global solution and (5) holds under suitable assumptions.

First, we need a lemma which is a Gronwall-type lemma for operator equations. 


\section{Lemma 15.1.}

$$
\dot{Q}=-T(t) Q+G(t), \quad Q(0)=Q_{0},
$$

where T, $G$ and $Q$ are bounded linear operator-functions in $H$. Assume that

$$
(T(t) h, h) \geq \varepsilon(t)\|h\|^{2} \quad \forall h \in H,
$$

where $\varepsilon(t)>0, \varepsilon(t) \in L_{l o c}^{1}(0, \infty)$. Then

$$
\|Q(t)\| \leq a^{-1}(t)\left\|Q_{0}\right\|+a^{-1}(t) \int_{0}^{t} a(s)\|G(s)\| d s, \quad a(t):=e^{\int_{0}^{t} \varepsilon(s) d s} .
$$

Proof. Let $g:=Q(t) h, b(t):=G h, h \in H$ is arbitrary, $h$ does not depend on $t$. Then (90) implies $\dot{g}=-T g+b(t)$. Multiply this equation by $g$, let $(g, g):=p^{2}(t)$, and get: $p \dot{p} \leq-\varepsilon(t) p^{2}+\|b(t)\| p$. Since $p(t) \geq 0$, one gets $p(t) \leq a^{-1}(t) p(0)+a^{-1}(t) \int_{0}^{t}\|b(s)\| a(s) d s$. Now take the supremum with respect to $h,\|h\|=1$, and get (92). Lemma 15.1 is proved.

Let us state the main result of this section.

Theorem 15.1. Assume (2), (3), and let $u_{0}$ and $Q_{0}$ be sufficiently close to $y$ and, respectively, to $\widetilde{A}^{-1}:=\left[F^{\prime}(y)\right]^{-1}$, where $F(y)=0$. Then problem (88)-(89) has a unique global solution $\left(\begin{array}{c}u(t) \\ Q(t)\end{array}\right), \exists u(\infty):=y, F(y)=0$, and $\|u(t)-y\| \rightarrow 0$ exponentially fast as $t \rightarrow \infty$.

Proof. Let $w:=u(t)-y$ and $\|w\|:=g$. Then $F(u)=\widetilde{A} w+K,\|K\| \leq$ $0.5 M_{2} g^{2}:=c_{0} g^{2}$, and (88) can be written as $\dot{w}=-Q[\widetilde{A} w+K]$. Let $\Lambda:=I-Q \widetilde{A}$. Then $\dot{w}=-w+\Lambda w-Q K$. Multiply this equation by $w$ and get

$$
g \dot{g}=-g^{2}+(\Lambda w, w)-(Q K, w) .
$$

Since $T(t) \geq c>0, c=$ const, Lemma 15.1 and equation (89) imply

$$
\|Q(t)\| \leq e^{-c t}\left\|Q_{0}\right\|+e^{-c t} \int_{0}^{t} M_{1} e^{s t} d t \leq\left\|Q_{0}\right\|+M_{1} c^{-1}:=c_{1},
$$

where $M_{1}$ is the constant from (2). Thus

$$
\|(Q K, w)\| \leq c_{1} c_{0} g^{3}:=k g^{3} .
$$

We prove below that

$$
\sup _{t \geq 0}\|\Lambda(t)\| \leq \lambda<1
$$


Thus (94) yields:

$$
\dot{g} \leq-\gamma g+k g^{2}, \quad 0<\gamma:=1-\lambda<1 .
$$

If $k g(0)<1$, then $(97)$ implies:

$$
g(t) \leq r e^{-\gamma t}, \quad r:=\frac{g(0)}{1-k g(0)} .
$$

Inequality $k g(0)<1$ holds if $k\left\|u_{0}-y\right\|<1$. This is the "closeness of $u_{0}$ to $y$ " condition. Inequality (98) shows that $u(t) \rightarrow y$ exponentially fast. The trajectory $\{u(t)\}_{t \geq 0} \in B\left(u_{0}, R\right)$ if $\int_{0}^{\infty}\|\dot{u}\| d t=\int_{0}^{\infty}\|\dot{w}\| d t \leq R$. Using (88), and the estimate: $\|F(u)\|=\|F(u)-F(y)\| \leq M_{1} g$, one gets $\|\dot{w}\| \leq\|Q\|\|F\| \leq c_{1} M_{1} g=c_{2} r e^{-\gamma t}$, where $c_{2}:=c_{1} M_{1}$. Thus $\int_{0}^{\infty}\|\dot{w}\| d t \leq$ $\frac{c_{2}}{\gamma} r \leq R$ if $r$ is sufficiently small, or (see (98)) if $u_{0}$ is sufficiently close to $y$. To complete the proof, one has to verify (96). One has

$$
\dot{\Lambda}=-\dot{Q} \widetilde{A}=T Q \widetilde{A}-A^{*} \widetilde{A}=-T \Lambda+A^{*}(A-\widetilde{A}) .
$$

Lemma 15.1 and (99) imply

$$
\|\Lambda(t)\| \leq e^{-c t}\left\|\Lambda_{0}\right\|+\int_{0}^{t} e^{-c(t-s)} M_{1} M_{2} r e^{-\gamma s} d s \leq\left\|\Lambda_{0}\right\|+C r
$$

where $C:=M_{1} M_{2} \sup _{t \geq 0} \int_{0}^{t} e^{-c t+(c-\gamma) s} d s$. If $\left\|\Lambda_{0}\right\|$ and $\left\|u_{0}-y\right\|$ are sufficiently small, then

$$
\left\|\Lambda_{0}\right\|+C r:=\lambda<1
$$

Theorem 15.1 is proved.

Remark 15.1. In the ill-posed case, when (3) fails, one can prove a similar result ( see $^{23}$ ).

\section{Equations with non-monotone operators}

Let us assume (2), but not (3), let $y$ solve equation (1), and let $A:=$ $F^{\prime}(u), T:=A^{*} A, T_{\varepsilon}:=T+\varepsilon I, A_{0}:=F^{\prime}(y)$, and $T_{0}:=A_{0}^{*} A_{0}$. Choose $\Phi=-T_{\varepsilon}^{-1}\left[A^{*} F(u)+\varepsilon\left(u-\tilde{u}_{0}\right)\right]$, where $\varepsilon=\varepsilon(t)$ satisfies conditions (21). Assume that $\tilde{u}_{0}$ is such that

$$
y-\tilde{u}_{0}=T_{0} z, \quad\|z\|<<1,
$$

where $\|z\|<<1$ means that $\|z\|$ is sufficiently small. This smallness requirement will be specified below. Assume also that $u_{0} \in B(y, R)$, and $R<<1$. Let $u=u(t)$ solve (4) with the above $\Phi$. 
Theorem 16.1. Under the above assumptions (5) holds for the solution to (4) with the above $\Phi$.

Remark 16.1. Theorem 16.1 states that the DSM with the above $\Phi$ is justified for solving equation (1) provided that (102) holds and $R<<1$. Condition $R<<1$ says that the initial approximation $u_{0}$ is sufficiently close to the solution $y$. This assumption is quite standard. Condition (102) means that the operator $T_{0}$ maps the punctured ball $\{z: 0<\|z\|<r\}$ of small radius $r>0$ onto a set which has a non-empty intersection with the set $\{w: 0<\|y-w\|<a\}$, where $a>0$ is some, possibly large, number. In the literature such type of assumption is called sometimes a source assumption, because it says that $y-\tilde{u}_{0}$ belongs to the range of $T_{0}$. The element $\tilde{u}_{0}$, such that the above assumption holds, exists if the range of $T_{0}$ is dense in a cone with vertex at $y$, or even when this range contains a segment issued from the element $y$. If $T_{0}$ is compact and has a non-zero eigenvalue: $T_{0} \phi=\lambda \phi,\|\phi\|=1, \lambda \neq 0$, then $\tilde{u}_{0}$ exists: for example one may take $\tilde{u}_{0}=y-s \phi$, where $s$ is sufficiently small. In this case $z=\frac{s}{\lambda} \phi$. Thus, assumption (102) is quite weak from the theoretical point of view. From the practical point of view it has a drawback: one does not have an algorithm for choosing suitable $\tilde{u}_{0}$.

Proof. Let us prove Theorem 16.1. Let $w:=u-y$ and $g:=\|w\|$. Using the Taylor's formula, one has:

$$
F(u)=F(u)-F(y)=A w+K, \quad\|K\| \leq 0.5 M_{2} g^{2} .
$$

Thus,

$$
\Phi=-T_{\varepsilon}^{-1}\left[T_{\varepsilon} w+A^{*} K+\varepsilon T_{0} z\right]=-w-T_{\varepsilon}^{-1} A^{*} K-\varepsilon T_{\varepsilon}^{-1} T_{0} z .
$$

One has $\left(\mathrm{cf}^{13}\right)$ :

$$
\left\|T_{\varepsilon}^{-1} A^{*}\right\| \leq \frac{1}{2 \sqrt{\varepsilon}}, \quad \varepsilon=\varepsilon(t), \quad \dot{u}=\dot{w}, \quad\left\|T_{0 \varepsilon}-T_{\varepsilon}\right\| \leq 2 M_{2} M_{1} g .
$$

Write $T_{\varepsilon}^{-1} T_{0}=\left(T_{\varepsilon}^{-1}-T_{0 \varepsilon}^{-1}\right) T_{0}+T_{0 \varepsilon}^{-1} T_{0}$. Note that $\left\|T_{0 \varepsilon}^{-1} T_{0}\right\| \leq 1$ and

$$
T_{\varepsilon}^{-1}-T_{0 \varepsilon}^{-1}=T_{\varepsilon}^{-1}\left(T_{0 \varepsilon}-T_{\varepsilon}\right) T_{0 \varepsilon}^{-1} .
$$

Multiply (4) (with the above $\Phi$ ) by $w$, use (103) and (104), and get the inequality:

$$
g \dot{g} \leq-g^{2}+\frac{c_{0} g^{3}}{\sqrt{\varepsilon}}+2 M_{2} M_{1}\|z\| g^{2}+\varepsilon\|z\| g,
$$


where $c_{0}:=M_{2} / 2$. Choose $\|z\| \leq \frac{1}{4 M_{1} M_{2}}$. Then, since $g \geq 0$, one gets:

$$
\dot{g} \leq-0.5 g+\frac{c_{0} g^{2}}{\sqrt{\varepsilon}}+\varepsilon\|z\| .
$$

Let us apply Theorem 7.1 to this differential inequality. One has $\gamma=0.5$, $\alpha=\frac{c_{0}}{\sqrt{\varepsilon}}$, and $\beta=\varepsilon\|z\|$. Choose $\mu=\mu(t)=\frac{p}{\sqrt{\varepsilon}}$. Then $\frac{\dot{\mu}}{\mu}=0.5 \frac{\dot{\varepsilon}}{\varepsilon} \leq \frac{1}{4}$, because of the assumption $\frac{|\dot{\varepsilon}|}{\varepsilon} \leq \frac{1}{2}$. Therefore the first condition (26) is satisfied if $p=8 c_{0}$. Since $\varepsilon(t) \leq \varepsilon(0)$, the second condition (26) is satisfied if $8 p \sqrt{\varepsilon(0)}\|z\| \leq 1$. This inequality holds if $\|z\|<<1$. The last condition (26) is satisfied if $\frac{p}{\sqrt{\varepsilon(0)}}\left\|u_{0}-y\right\|<1$. This condition is satisfied if $R<<1$. Thus, Theorem 16.1 is proved.

One can derive a stopping rule using the ideas presented at the end of Section 6. If $F(u):=B(u)-f$, noisy data $f_{\delta}$ are given, such that $\left\|f_{\delta}-f\right\| \leq \delta$, and $w_{\delta}:=u_{\delta}-y$, where $u_{\delta}=u_{\delta}(t)$ solves problem (4) with $\Phi$ as in Theorem 16.1 and $f_{\delta}$ replacing $f$, then for $g_{\delta}:=g_{\delta}(t):=\left\|w_{\delta}\right\|$ one derives, as in the proof of Theorem 16.1, the following inequality:

$$
\dot{g}_{\delta} \leq-g_{\delta}+\frac{c_{0} g_{\delta}^{2}}{\sqrt{\varepsilon}}+\frac{\delta}{2 \sqrt{\varepsilon}}+\varepsilon\|z\|+2 M_{1} M_{2}\|z\| g_{\delta} .
$$

Choose $\|z\| \leq \frac{1}{4 M_{1} M_{2}}$. This is possible due to the assumption $\|z\|<<1$. Then inequality (105) can be written as:

$$
\dot{g}_{\delta} \leq-0.5 g_{\delta}+\frac{c_{0} g_{\delta}^{2}}{\sqrt{\varepsilon}}+\frac{\delta}{2 \sqrt{\varepsilon}}+\varepsilon\|z\| .
$$

Use Theorem 7.1 and choose $\mu=\frac{p}{\sqrt{\varepsilon}}, \varepsilon=\varepsilon(t)$. Then $\frac{\dot{\mu}}{\mu}=0.5 \frac{|\dot{\varepsilon}|}{\varepsilon}$. Since $\frac{|\dot{\varepsilon}|}{\varepsilon} \leq \frac{1}{2}$, one has the first condition (26) satisfied if $p=\frac{8 c_{0}}{3}$. The second condition (26) is satisfied if $\frac{\delta}{2 \sqrt{\varepsilon}}+\varepsilon\|z\| \leq \frac{3 \sqrt{\varepsilon}}{8 p}$. This condition is satisfied because of the assumption $\|z\|<<1$ and $\frac{\delta}{2 \sqrt{\varepsilon}}$ is sufficiently small, that is, $t$ is not too large, $t \leq t_{\delta}$, where the choice of $t_{\delta}$ is discussed below. The third inequality $(26)$ is of the form: $\frac{p}{\sqrt{\varepsilon(0)}}\left\|u_{0}-y\right\|<1$. It is satisfied if $\left\|u_{0}-y\right\|<<1$. This condition holds because of the assumption $R<<1$. Applying Theorem 7.1 to inequality (106), one gets the inequality $g_{\delta}(t) \leq$ $c \varepsilon(t)$, provided $t \leq t_{\delta}$. The stopping time $t_{\delta} \rightarrow \infty$ as $\delta \rightarrow 0$. For example, one can take as $t_{\delta}$ the solution to the equation $\delta=\varepsilon(t)$, which has a unique solution because $\varepsilon(t)$ is monotonically tends to zero as $t \rightarrow \infty$. This choice of $t_{\delta}$ is easy to use numerically. 


\section{References}

1. R. Airapetyan, A. G. Ramm, A. B. Smirnova, Continuous analog of GaussNewton method Math. Models and Methods in Appl. Sci., 9, N3, (1999), 463-474.

2. R. Airapetyan, A. G. Ramm, Dynamical systems and discrete methods for solving nonlinear ill-posed problems, Appl.Math.Reviews, vol. 1, Ed. G. Anastassiou, World Sci. Publishers, 2000, pp.491-536.

3. R. Airapetyan, A. G. Ramm, A. B. Smirnova, Continuous methods for solving nonlinear ill-posed problems, in the book "Operator theory and applications", Amer. Math. Soc., Fields Institute Communications, Providence,RI, 2000, pp. 111-138. (editors A.G.Ramm, P.N.Shivakumar, A.V.Strauss).

4. A.B.Bakushinsky, and M.Yu. Kokurin, Iterative methods for ill-posed operator equations with smooth operators, YRSS, Moscow, 2002.(in Russian)

5. M.K. Gavurin, Nonlinear functional equations and continuous analogies of iterative methods, Izv. Vuzov. Ser. Matematika, 5, (1958), 18-31.

6. V. Ivanov, V. Vasin, V. Tanana, Theory of linear ill-posed problems, Nauka, Moscow, 1978. (in Russian)

7. B. Kaltenbacher, A. Neubauer, A. G. Ramm, Convergence rates of the continuous regularized Gauss-Newton method, Jour. Inv. Ill-Posed Probl., 10, N3, (2002), 261-280.

8. Kamke E., Differentialgleichungen. Lösungmethoden und Lösungen, Chelsea, New York, 1974.

9. J.Ortega, W.Rheinboldt, Iterative solution of nonlinear equations in several variables, SIAM, Philadelphia, 2000.

10. A. G. Ramm, Inverse problems, Springer, New York, 2005.

11. A. G. Ramm, On the discrepancy principle, Nonlinear Functional Anal. and Applic., 8, N2, (2003), 307-312.

12. DSM for ill-posed equations with monotone operators, Comm. in Nonlinear Sci. and Numer. Simulation, 10, N8, (2005),935-940.

13. _ Dynamical systems method for solving operator equations, Communic. in Nonlinear Sci. and Numer. Simulation, 9, N2, (2004), 383-402.

14. In Inequalities for solutions to some nonlinear equations, Nonlinear Functional Anal. and Applic., 9, N2, (2004), 233-243.

15. Discrepancy principle for the dynamical systems method, Communic. in Nonlinear Sci. and Numer. Simulation, 10, N1, (2005), 95-101

16. Dynamical systems method for solving nonlinear operator equations International Jour. of Applied Math. Sci., 1, N1, (2004), 97-110.

17. Dynamical systems method and surjectivity of nonlinear maps, Communic. in Nonlinear Sci. and Numer. Simulation, 10, N8, (2005), 931934 .

18. Dynamical systems method (DSM) for unbounded operators, Proc. Amer. Math. Soc., 134, N3, (2006), 1059-1063.

19. A nonlinear singular perturbation problem (submitted)

20. A new discrepancy principle, Jour. Math. Anal. Appl., 310, (2005), 342-345. 
21. Dynamical systems method and a homeomorphism theorem, Amer. Math. Monthly, (2006) (to appear)

22. _ Dynamical systems method for nonlinear equations in Banach spaces Comm. Nonlin Sci. and Numer. Simul., 11, N3, (2006), 306-310.

23. A. G. Ramm, A. B. Smirnova, Continuous regularized Gauss-Newton-type algorithm for nonlinear ill-posed equations with simultaneous updates of inverse derivative, Intern. Jour. of Pure and Appl Math., 2, N1, (2002), 23-34.

24. A. G. Ramm, A. B. Smirnova, On stable numerical differentiation, Mathem. of Computation, 70, (2001), 1131-1153.

25. A. G. Ramm, A. B. Smirnova, Stable numerical differentiation: when is it possible? Jour. Korean SIAM, 7, N1, (2003), 47-61.

26. A. G. Ramm, A. B. Smirnova, A numerical method for solving nonlinear ill-posed problems, Numerical Funct. Anal. and Optimiz., 20, N3, (1999), 317-332.

27. Tikhonov A., Leonov, A., Yagola, A., Nonlinear ill-posed problems, vol. 1,2, Chapman and Hall, London, 1998. 